\title{
Mosquito-Bacteria Symbiosis: The Case of Anopheles gambiae and Asaia
}

\author{
Claudia Damiani • Irene Ricci • Elena Crotti • Paolo Rossi • Aurora Rizzi • \\ Patrizia Scuppa • Aida Capone • Ulisse Ulissi • Sara Epis • Marco Genchi • \\ N'Fale Sagnon • Ingrid Faye • Angray Kang • Bessem Chouaia • Cheryl Whitehorn • \\ Guelbeogo W. Moussa • Mauro Mandrioli • Fulvio Esposito • Luciano Sacchi • \\ Claudio Bandi • Daniele Daffonchio • Guido Favia
}

Received: 3 December 2009 / Accepted: 8 June 2010 / Published online: 23 June 2010

(C) Springer Science+Business Media, LLC 2010

\begin{abstract}
The symbiotic relationship between Asaia, an $\alpha$-proteobacterium belonging to the family Acetobacteriaceae, and mosquitoes has been studied mainly in the Asian malaria vector Anopheles stephensi. Thus, we have investigated the nature of the association between Asaia and the major Afro-tropical malaria vector Anopheles gambiae. We
\end{abstract}

Claudia Damiani, Irene Ricci and Elena Crotti have equally contributed to this work.

C. Damiani $\cdot$ I. Ricci $\cdot$ P. Rossi $\cdot$ P. Scuppa $\cdot$ A. Capone $\cdot$

U. Ulissi $\cdot$ S. Epis $\cdot$ F. Esposito $\cdot$ G. Favia $(\triangle)$

Scuola di Bioscienze e Biotecnologie,

Università degli Studi di Camerino,

Via Gentile III da Varano,

62032 Camerino, Italy

e-mail: guido.favia@unicam.it

E. Crotti $\cdot$ A. Rizzi $\cdot$ B. Chouaia $\cdot$ D. Daffonchio

Dipartimento di Scienze e Tecnologie Alimentari

e Microbiologiche, Università degli Studi di Milano,

Via Celoria 2,

20133 Milan, Italy

M. Genchi $\cdot$ L. Sacchi

Dipartimento di Biologia Animale,

Università degli Studi di Pavia,

P.zza Botta 9,

27100 Pavia, Italy

N. Sagnon $\cdot$ G. W. Moussa

Centre National de Recherche et de Formation sur le Paludisme

(CNRFP),

01B.P.2208,

Ouagadougou 01, Burkina Faso

\section{Faye}

Department of Genetics Microbiology and Toxicology,

Stockholm University,

Svante Arrhenius v. 16 E-F,

SE-106 91 Stockholm, Sweden have isolated Asaia from different wild and laboratory reared colonies of A. gambiae, and it was detected by PCR in all the developmental stages of the mosquito and in all the specimens analyzed. Additionally, we have shown that it localizes in the midgut, salivary glands and reproductive organs. Using recombinant strains of Asaia expressing
M. Mandrioli

Dipartimento di Biologia,

Università degli Studi di Modena e Reggio Emilia,

Via Campi 213/D,

41125 Modena, Italy

\section{Bandi}

Dipartimento di Patologia Animale, Igiene e Sanità Pubblica

Veterinaria, Università degli Studi di Milano,

Via Celoria 10,

20133 Milan, Italy 
fluorescent proteins, we have demonstrated the ability of the bacterium to colonize $A$. gambiae mosquitoes with a pattern similar to that described for A. stephensi. Finally, fluorescent in situ hybridization on the reproductive tract of females of $A$. gambiae showed a concentration of Asaia at the very periphery of the eggs, suggesting that transmission of Asaia from mother to offspring is likely mediated by a mechanism of egg-smearing. We suggest that Asaia has potential for use in the paratransgenic control of malaria transmitted by $A$. gambiae.

\section{Introduction}

To date malaria is still a major cause of death in Sub-Saharan African children and the number of human cases is increasing world-wide, thus the development of novel approaches to fight this deadly disease is urgently needed [2]. Malaria parasite requires a mosquito vector for transmission, and recent technical advances in vector biology have lead to a new strategy to combat malaria, by the means of the genetic modification of the mosquito to reduce its vectorial competence [12, 15]. More recently, a great deal of attention has been devoted to the development of a novel approach, based on the manipulation of bacterial symbionts inhabiting the midgut lumen of the vector, in order to generate symbionts producing anti-plasmodial effector molecules inside mosquitoes [19]. Indeed, the midgut hosts the oocyst, the most vulnerable stage of Plasmodium development, which represents a bottleneck of the malaria cycle since only few oocysts develop inside a single mosquito (from one to a few dozen depending on the mosquito species) [1].

The use of bacterial manipulation to interfere with the vectorial competence of the mosquito is favoured by both technical and ethical considerations [16]. Genetic manipulation of bacteria is simpler and faster than genetic manipulation of mosquitoes, bacteria are much easier to introduce into mosquito populations and can be produced easily and cheaply in large quantities. Furthermore, the introduction of modified bacteria into a mosquito population will bypass genetic barriers of reproductively isolated mosquito populations which often occur in endemic malaria regions [11] and, differently from the case for transgenic insect release, regulations on the release of bacteria in the environment already exist, and these rules could guide the development of bacteria-based protocols for malaria control.

We have initiated a study to identify symbiotic bacteria inhabiting the midgut of mosquitoes, with the purpose of selecting symbionts potentially useful for the development of paratransgenic strategies for malaria control [14, 18]. Recently, we identified an acetic acid $\alpha$-proteobacterium belonging to the genus Asaia [22] that is stably associated with midgut, salivary glands and reproductive organs of the
Asian malaria vector Anopheles stephensi [9, 10]. Asaia has been isolated from $A$. stephensi and proven to be easily cultivable and transformable, and modified strains of Asaia, expressing green fluorescent protein (GFP) or red fluorescent protein (DsRed), efficiently colonize recipient mosquitoes [4]. Asaia is horizontally transmitted to members of mosquito populations by co-feeding and mating, and by maternal and paternal vertical transmission routes [4, 9]. Taking into consideration all these features, Asaia can be considered a promising agent for the paratransgenic control of malaria vectors.

Most of the previous work on the symbiotic relationship between Asaia and mosquitoes was performed on labreared specimens belonging to the species $A$. stephensi. Here we present a detailed study on the association between Asaia and Anopheles gambiae, which is widespread in Africa and is the most anthropophilic and efficient malaria vector in the world. This study included field collected specimens and investigated the ability of genetically modified strains of Asaia to colonize A. gambiae.

\section{Materials and Methods}

\section{Mosquitoes}

In this study, four different sources of mosquitoes belonging to the species $A$. gambiae were employed; in particular, we used three lab-reared colonies from the insectaries of (1) University of Camerino (UNICAM), (2) London School of Hygiene and Tropical Medicine (LSHTM), (3) University of Stockholm (SU) and (4) a wild African specimens collection:

1. Mosquitoes from UNICAM (Camerino, Italy) were obtained from the Centre National de Recherche et de Formation sur le Paludisme (CNRFP) in Ouagadougou (Burkina Faso, West Africa) and descend from a wild colony. The samples used in this study have been reared for about 2 years in the UNICAM insectary, where they were maintained at standard lighting conditions of $12 \mathrm{~h}$ light and $12 \mathrm{~h}$ dark, a relative humidity of $80 \pm 5 \%$ and temperature of $30^{\circ} \mathrm{C}$, in aseptic conditions during both the developmental and adult stages. The larvae were grown in tanks filled with culture water containing sterile minced commercial mouse food and adults were fed on mouse blood and a 5\% glucose solution;

2. Mosquitoes from LSHTM (London, UK) belong to a G3 strain from McCarthy Island, The Gambia, West Africa. The samples used in this study have been reared for 34 years in the LSHTM insectary, where they were maintained in standard lighting conditions of $12 \mathrm{~h}$ light and $12 \mathrm{~h}$ dark, a relative humidity of $60-70 \%$ and 
temperature of $30^{\circ} \mathrm{C}\left( \pm 5^{\circ} \mathrm{C}\right)$, in aseptic conditions during both the developmental and adult stages. The larvae were fed on a diet of ground baby muesli produced by Heinz Babyfoods and adults on defibrinated horse blood and a $10 \%$ glucose solution;

3. Mosquitoes from SU (Stockholm, Sweden) belong to a G3 strain from (MR4)/ATCC (Manassas, Virginia). The samples used in this study have been reared for 1.5 years in the SU insectary, where they were maintained at standard lighting conditions of $12 \mathrm{~h}$ light and $12 \mathrm{~h}$ dark, a relative humidity of $80 \%$ and temperature of $27^{\circ} \mathrm{C}$, in aseptic conditions during both the developmental and adult stages. The larvae were fed on fish food $\left(\right.$ Tetramin $\left.^{\circledR}\right)$ and adults on human blood and $10 \%$ sucrose solution;

4. Field collected mosquitoes were indoor captured by aspirator tubes in Goundry, a village close to Ouagadougou. Soon after the capture, species identification has been performed on morphological bases. For further microbiological and molecular analyses, most of the specimens were sacrificed immediately after the transport from the field in the lab, while, few gravid females were maintained alive and brought in the insectary of CNRFP to obtain the F1 generation.

\section{Polymerase Chain Reaction Analysis for Asaia Detection}

Mosquitoes metagenome extraction was performed on whole insects (pre- and adults) and organs/tissues as previously described [8]. Dissections were carried out under a stereomicroscope on a slide rinsed with a drop of sterile $1 \times$ PBS using sterile needles. To avoid crosscontamination among tissues, just a single organ (salivary glands, gut or reproductive organ) was recovered from an individual mosquito. DNA extraction was performed under a sterile cabinet (Bioair, Euroclone, Italy). Purified DNA was used as template in polymerase chain reaction (PCR) amplification experiments with Asaia specific primers Asafor (5'-GGCGCGTAGGCGGTTTACAC-3') and Asa-rev (5'-AGCGTCAGTAATGAGCCAGGTT-3'), targeting 16S rRNA gene and amplifying a product of $186 \mathrm{bp}$ [9]. Reaction mixtures were prepared in $25 \mu$ using: 1 unit of Super $\mathrm{AB}$ Taq DNA polymerase (AB analitica, Italy), $0.25 \mathrm{mM}$ dNTPs, $2.5 \mathrm{mM} \mathrm{MgCl}{ }_{2}, 1 \times$ Taq polymerase buffer, $1 \mu \mathrm{M}$ each primer, and $50 \mathrm{ng}$ DNA. Reactions were run for $5 \mathrm{~min}$ at $94^{\circ} \mathrm{C}$ and cycled 30 times through $30 \mathrm{~s}$ at $94^{\circ}$ $\mathrm{C}, 30 \mathrm{~s}$ at $62^{\circ} \mathrm{C}$ and $30 \mathrm{~s}$ at $72^{\circ} \mathrm{C}$. Finally, reactions were kept for $8 \mathrm{~min}$ at $72^{\circ} \mathrm{C}$. PCR products were then resolved in an $1 \%$ agarose gel stained with ethidium bromide. The overall PCR screen is summarized in Table 1. As negative control, pure genomic DNA $(10 \mathrm{ng})$ from Acetobacter aceti and Gluconobacter sp. were used: no amplification was observed. Furthermore, some of the amplicons obtained, have been sequenced confirming to be Asaia-specific (accession number AM404260). Larval breeding water and feeding sucrose solution were also analyzed to exclude environmental contamination that would have affected the overall screening.

\section{Transmission Electron Microscopy}

Laboratory reared A. gambiae (UNICAM strain) adult mosquitoes were dissected in a sterile saline solution to separate the female salivary glands and both male and female midgut and the reproductive system. Semithin sections for light microscopy and thin sections for transmission electron microscopy (TEM) were prepared and examined as described in [20].

Asaia Isolation, Strains Identification and Transformation

Asaia sp. was isolated from A. gambiae mosquitoes belonging to both lab-reared and field collected specimens from respectively the colony reared in UNICAM insectary, the G3 strain reared in LSHTM insectary, and the Goundry field collection ( $\mathrm{F} 0$ and $\mathrm{F} 1$ ); the isolates were respectively named AG1.5Aa, AGL, AGF0 and AGF1. For bacterial isolation we started from fresh adult mosquito homogenates by a pre-enrichment step in liquid medium ( $\mathrm{pH} 3.5)$, then plated on agar medium added with calcium carbonate as previously described for isolation of Asaia from $A$. stephensi [9]. Asaia sp. isolates were identified by morphological analysis of the colonies and formation of carbonate dissolution haloes in agar plates, subsequently their identification were confirmed by 16S rRNA gene sequencing. Briefly, colonies were picked and DNA was extracted using a commercial kit (Qiagen, Germany). PCR products obtained using universal bacterial primers, $27 \mathrm{~F}$ (5'-TCGACATCGTTTACGGCGTG-3') and 1492R (5'CTACGGCTACCTTGTTACGA-3'), targeted on the $16 \mathrm{~S}$ rRNA gene, were sequenced. The sequences obtained (accession numbers FN814275, FN821396, FN821397 and FN821398) and other 16 sequences obtained from the data bases were aligned using the multiple sequence alignments algorithm implemented in MUSCLE [7], setting parameters as default. The alignment was checked manually. Trees representing the relationships between the sequences included in the alignment were constructed using both the neighbour joining (NJ) algorithm after different corrections (using Treecon 1.3b [21]) and the maximum likelihood (using phyML 3.0; www.atgc-montpellier.fr/ phyml/). The GFP-tagged strain AGF0(pHM2-gfp), was generated by the transformation of Asaia sp. isolated from the A. gambiae African field collected specimens, following the protocol already used for the GFP-tagged strain obtained from A. stephensi SF2.1(pHM2-gfp) [9]. Both recombinant strains have been modified by transformation 
Table 1 Results summary of PCR screens targeting 16S rRNA for the detection of wild-type Asaia in different samples of A. gambiae

\begin{tabular}{|c|c|c|c|}
\hline \multicolumn{2}{|l|}{ A. gambiae strains } & \multirow{2}{*}{$\begin{array}{l}\text { Stage/tissue } \\
\text { Adults }\end{array}$} & \multirow{2}{*}{$\begin{array}{l}\text { PCR tested samples }\left(\mathrm{n}^{\circ} \text { of sequenced amplicons) }\right. \\
85 \% ; 15 \lesssim(20)\end{array}$} \\
\hline Wild mosquitoes & Goundry F0 & & \\
\hline & Goundry F1 & Adults & $25 ;$; $25 \lesssim(10)$ \\
\hline \multirow[t]{11}{*}{ Lab-reared mosquitoes } & LSHTM strain & Adults & 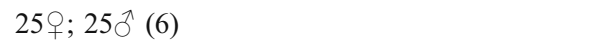 \\
\hline & SU strain & Adults & 25 우 $25 \hat{\jmath}$ \\
\hline & UNICAM strain ${ }^{(b)}$ & Adults & 25 ㅇ; $250 \hat{~(10) ~}$ \\
\hline & & Eggs & $50(5)$ \\
\hline & & Larvae & $50(7)$ \\
\hline & & Pupae & $50(7)$ \\
\hline & & † salivary glands & $25(2)$ \\
\hline & & $q$ guts & $40(4)$ \\
\hline & & q reproductive systems & $30(3)$ \\
\hline & & $\widehat{\partial}$ guts & $40(4)$ \\
\hline & & $\widehat{\delta}$ reproductive systems & $30(3)$ \\
\hline
\end{tabular}

\footnotetext{
${ }^{\mathrm{a}}$ All of the tested samples resulted positives to the Asaia-specific PCR analysis and some obtained amplicons were randomly sequenced to confirm their homology. Test specificity for Asaia was confirmed on pure genomic DNA from A. aceti and Gluconobacter sp.

${ }^{\mathrm{b}}$ Mosquito belonging to three different generations have been analyzed (sample size per each generation is shown). Larval breeding water and adults feeding sucrose solution were used as negative control in all the amplification runs
}

with the pHM2 plasmid carrying both the $g f p$ and kanamycin resistance gene cassettes.

\section{Mosquitoes Colonization By Asaia Expressing GFP and DsRed}

For the colonization experiments of $A$. gambiae (UNICAM strain) the following three recombinant Asaia sp. were employed: SF2.1(pHM2-gfp) and SF2.1(DsRed), both belonging to the strain SF2.1 previously isolated from $A$. stephensi $[4,9]$, and AGF0(pHM2-gfp) isolated from the Goundry wild collection of $A$. gambiae. The DsRed-tagged bacteria were constructed by insertion of the $d s R e d$ gene into the chromosome of Asaia sp. strain SF2.1 and used in the colonization experiments as stable recombinant which do not need antibiotic selection [4, 17]. All recombinant bacteria were grown $24 \mathrm{~h}$ at $30^{\circ} \mathrm{C}$ in GLY medium $\left(25 \mathrm{~g}^{-1}\right.$ glycerol, $10 \mathrm{~g}^{-1}$ yeast extract, $\mathrm{pH} \mathrm{5)}$ and cells were harvested by centrifugation, washed three times in $0.9 \%(w / v) \mathrm{NaCl}$ and adjusted to $10^{8}$ cells ml ${ }^{-1}$ in $30 \mathrm{ml}$ of sterile $\mathrm{H}_{2} \mathrm{O}$ /sugar $5 \%$ $(w / v)$ sucrose solution. In order to obtain the colonization of the insects, sucrose solutions containing the fluorescent bacteria $\left(10^{8}\right.$ cells $\left.\mathrm{ml}^{-1}\right)$ were offered to the mosquitoes on a sterile cotton pad. Different sets of experiments have been performed including three cages $(\mathrm{A}, \mathrm{B}, \mathrm{C})$ each containing around 100 adult mosquitoes belonging to the UNICAM colony. In each cage insects were allowed to feed one of the three modified Asaia sp.: cage A, AGF0(pHM2-gfp); cage B, SF2.1(pHM2-gfp); cage C, SF2.1(DsRed). Because the GFP-tagged Asaia strains carry the kanamycin resistance gene cassette for the selection, the mosquitoes supplemented with GFP-labelled bacteria (cages A and B) were maintained with sucrose diet plus kanamycin $\left(100 \mu \mathrm{g} \mathrm{ml}^{-1}\right)$ while mosquitoes supplemented with DsRed-tagged bacteria fed on their usual (kanamycin-free) diet (cage C). Bacterial supplemented diet was prolonged $48 \mathrm{~h}$, then mosquitoes were left at least two days without any bacterial feed before collection of the organs. Dissections were carried out as described in the section 2.2. Sampling for microscopy analysis was performed at different intervals up to 20 days post bacterial consumption. Guts, salivary glands and reproductive organs were examined for GFP- or DsRedtagged recombinant bacteria detection with an IX71 fluorescent microscope (Olympus, Melville, NY). A given organ was considered "positively" colonized after detection of at least five fluorescent bacteria. All tissues were fixed with $4 \%$ paraformaldehyde for $10 \mathrm{~min}$ at $4^{\circ} \mathrm{C}$, with the exception of salivary glands which were analyzed on fresh prepared slides. The slides were then mounted in glycerol-PBS for analysis.

\section{Fluorescence In Situ Hybridization}

Ovaries of gravid A. gambiae female mosquitoes (UNICAM strain) dissected in sterile conditions (see 2.2) were analyzed few days after blood meal to detect natural occurring Asaia sp. in the eggs. In particular we used two Asaia sp. specific probes targeting the 16S rRNA gene, Asaia1.FCy3 (5'-GTGTAAACCGCCTACGCGCC-3') and Asaia2.FCy3 (5'-ATGGATAGATCCCTACGCGA-3') labelled at the $5^{\prime}$ end with the fluorochrome $\mathrm{Cy} 3$ (indocarbocyanine, absorption/emission at 550/570 nm), that have 
been designed by ClustalW (http://align.genome.jp/) alignment among members of the Acetobacteraceae family. Specificity of Asaia-targeting oligonucleotide probes has been validated by ProbeMatch tool (http://rdp.cme.msu. edu/probematch/search.jsp). Additional probe EUB338 (5'GCTGCCTCCCGTAGGAGT-3'), routinely used as a universal bacterial probe [5], has been employed as bacterial positive control. Probe EUB338 was labelled at the $5^{\prime}$ end with digoxigenin (DIG) in order to be recognized by an anti-DIG antibody coupled with fluorescein isothiocyanate (FITC, absorption/emission at 494/520 nm). Before hybridization dissected tissues were fixed in $4 \%$ paraformaldehyde for $5 \mathrm{~min}$ at $4^{\circ} \mathrm{C}$ and washed in $1 \times$ PBS. Dissected organs were incubated for $15 \mathrm{~min}$ at $37^{\circ} \mathrm{C}$ with a $10 \mathrm{mg} \mathrm{ml}^{-1}$ pepsin solution and washed twice in a solution of $1 \times$ PBS containing $1 \%$ Tween 20 and once in $1 \times$ PBS for $5 \mathrm{~min}$ at room temperature. Hybridization was carried out in the dark for $3 \mathrm{~h}$ at $43^{\circ} \mathrm{C}$, with $100 \mu \mathrm{l}$ of hybridization buffer $(2 \times \mathrm{SSC}, 30 \%$ formamide, dextran sulphate $1 \%$, $10 \mathrm{ng} \mathrm{ml}^{-1}$ probes). After hybridization, dissections were washed in: $200 \mu \mathrm{l}$ of washing buffer ( $2 \times \mathrm{SSC}, 60 \%$ formamide and dextran sulphate $1 \%$ ) for $15 \mathrm{~min}$ at $43^{\circ} \mathrm{C}$, $500 \mu \mathrm{l}$ of $0.1 \times \mathrm{SSC}$ and twice in $200 \mu \mathrm{l}$ of $1 \times \mathrm{SSC}$ for $10 \mathrm{~min}$ at room temperature. Followed an incubation of $30 \mathrm{~min}$ at $37^{\circ} \mathrm{C}$ with anti-DIG antibody (1:300; Roche) and two final washes in $1 \times$ PBS for $10 \mathrm{~min}$ at room temperature. Then $100 \mathrm{ng}$ of DAPI were added, and incubated for $5 \mathrm{~min}$ at room temperature. After two washes in $1 \times$ PBS at room temperature samples were mounted in antifading medium and observed using a laser-scanning confocal microscope SP2-AOBS (Leica). The absence of autofluorescence has been evaluated by fluorescence in situ hybridization (FISH) control experiments involving treatment of slides in absence of Asaia- or Eubacteria-specific probes. Negative controls were carried out by hybridization with the Cy3-tagged Asaia-specific probes in absence of the probe EUB338 and vice versa. To test that the oligonucleotide probes did not target unspecifically cell components, we also carried out hybridisation experiment after RNase treatment $(10 \mu \mathrm{g} / \mathrm{ml}) 10 \mathrm{~min}$ at $37^{\circ} \mathrm{C}$. Distribution of the fluorescence has been evaluated using the Interactive 3D Surface Plot plugin (freely available at the address http:// rsbweb.nih.gov/ij/plugins/surface-plot-3d.html) of the software for image processing and analysis ImageJ.

\section{Results}

Identification of Asaia in A. gambiae Mosquitoes: Tissue Specificity and Developmental Stage Analysis

By PCR analysis targeting the 16S rRNA gene of Asaia, we were able to detect its DNA in the metagenome of $A$. gambiae mosquitoes coming from the three lab-reared colonies (UNICAM, SU and LSHTM) and from the wild Goundry African collection (Table 1). The presence of Asaia has been detected in all the tested specimens, analysing at least 50 adults for each of the reared mosquito lines and 100 individuals from the wild collection. Additionally, from the field-collected mosquitoes, Asaia was also detected in all the tested samples (50 mosquitoes) belonging to the F1 generation obtained in the lab from a few wild gravid females. Maintenance of the mosquitoes in the UNICAM insectary allowed us to perform further analyses to better characterize the bacterial localization inside the insect body and its profile of distribution in the vector population. By the same PCR assay, we searched Asaia in the developmental stages as well as in different organs and tissues of the mosquito. We detected the bacterium in all the pre-adult stages (eggs, L1-L4 larvae, pupae) as well as in the midgut, salivary glands and gonads of adult mosquitoes, in the whole tested sample from several reared generations. It was indeed its presence in the reproductive apparatus of $A$. gambiae that was suggestive for a specific association of the microorganism, rather than for an occasional presence related for example to food consumption, as further supported by the failure of Asaia detection in the larval breeding water and in the adult feeding sucrose solution. Moreover, the evidence that Asaia is harboured in $100 \%$ of the analyzed wild and reared mosquitoes (both males and females), likely reflects the very effective (horizontal and vertical) transmission routes of these bacteria, already described in A. stephensi $[4,9]$. All these findings strongly indicate that Asaia, previously known as the dominant bacterium in the microbiota of $A$. stephensi is also tightly associated with $A$. gambiae.

TEM observations on organs from $A$. gambiae adult mosquitoes (UNICAM strain) confirmed tissue localization indicated by PCR analysis. Cells presenting the morphological signatures of Asaia, i.e. a bright filamentous nucleoid region and an external slime layer lining the cell surface [9], could be clearly seen in the mosquito midgut (Fig. 1).

Isolation of Asaia from A. gambiae and Colonization of Mosquito Body By Modified Strains of Asaia

We were able to isolate bacteria of the genus Asaia from four populations of $A$. gambiae: UNICAM insectary, LSHTM insectary, Goundry F0 and Goundry F1. Asaia sp. isolates (AG1.5Aa, AGL, AGF0 and AGF1, respectively) were characterized at the molecular level by full length amplification and sequencing of the 16S rRNA gene. The sequences obtained revealed limited variation between Asaia harboured by mosquitoes from the different populations of $A$. gambiae (0-5 nucleotide differences). Figure 2 presents an example of tree, obtained by a distance matrix-based method (see figure 
Figure 1 TEM micrographs of an A. gambiae (UNICAM strain) adult female gut. Midgut lumen showing large amount of Asaia (a). Asterisks indicate filamentous structures in the nucleoid region. An extracellular matrix (arrow) with a fibrillar nature can be observed (b)
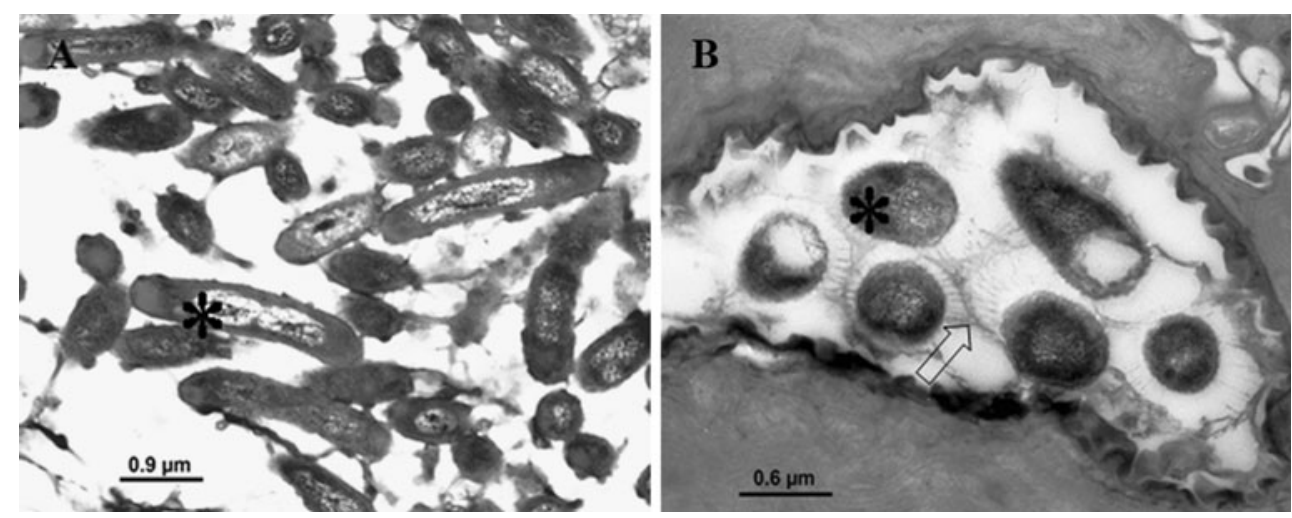

legend); trees obtained using different methodologies (different corrections, or maximum likelihood; see "Materials and Methods)" showed the same overall topology. The phylogenetic trees based on 16S rRNA sequences revealed the clustering of Asaia from wild-collected F0 and F1 mosquitoes, from the strain held in Camerino (UNICAM) and from a G3-derived $A$. gambiae strain (this last sequence is available in the data-bases; FJ816021.1). There is thus evidence that $A$. gambiae mosquitoes originated from two different stocks (field captured in Burkina Faso for F0, F1 and UNICAM; MR4, American-type culture collection Manassas, Va USA for G3) harbour closely related Asaia. However, A. gambiae LSHTM, which also derive from a G3 strain (McCarthy Island, The Gambia, West Africa) was shown to harbour an Asaia that clusters with Asaia from Aedes aegypti, in a different part of the tree. In addition, the trees generated also showed clustering of sequences of Asaia from mosquitoes with sequences from environmental samples. In terms of the possible affiliation to the described species of Asaia, isolates AG1.5Aa, AGF0 and AGF1 clustered with Asaia krungthepensis, while AGL clustered with Asaia bogorensis as well as with strain SF2.1 previously isolated from A. stephensi [9]. It is anyway out of the scope of the current work to refer to the Asaia strains detected in mosquitoes with species names.

Asaia sp. isolated from the wild mosquitoes (F0) was manipulated to obtain the recombinant green fluorescent bacterial strain AGF0(pHM2-gfp), that was then used in the mosquito colonization experiments along with SF2.1(pHM2$g f p$ ) and SF2.1(DsRed) (Table 2), previously generated by the modifications of the Asaia sp. strain SF2.1 [4, 9]. Two GFP-tagged Asaia strains, SF2.1(pHM2-gfp) or AGF0 (pHM2-gfp), were supplemented into the sugar meal along with kanamycin and provided to mosquitoes belonging to the UNICAM reared colony of $A$. gambiae (cages A and B, respectively). With respect to the strain Asaia sp. AGF0 (pHM2-gfp) the colonization experiments were performed in triplicate on mosquitoes of different generations and sampled at different intervals for up to 20 days after initial exposure to the bacteria. Mosquitoes were dissected and guts, salivary glands and reproductive organs were analyzed by fluorescence microscopy to detect the recombinant bacteria. All of
Figure 2 Representative tree based on partial 16S rRNA gene sequences $(>1,000 \mathrm{bp})$ from Asaia spp. The tree was generated using Treecon 1.3b using the neighbour joining $(\mathrm{NJ})$ method, after Kimura correction (transition/transversion ratio was estimated from the data). Insertions/deletions were not taken into account. The number at the nodes are the bootstrap confidence values obtained after 100 replications; the first number is the bootstrap value for the $\mathrm{NJ}$ tree, while the second number is the value for the same node after maximum likelihood analysis. Only bootstrap values $>50$ are represented. The EMBL/GenBank accession number for each sequence is indicated. Bar: 0.1 inferred substitutions per site

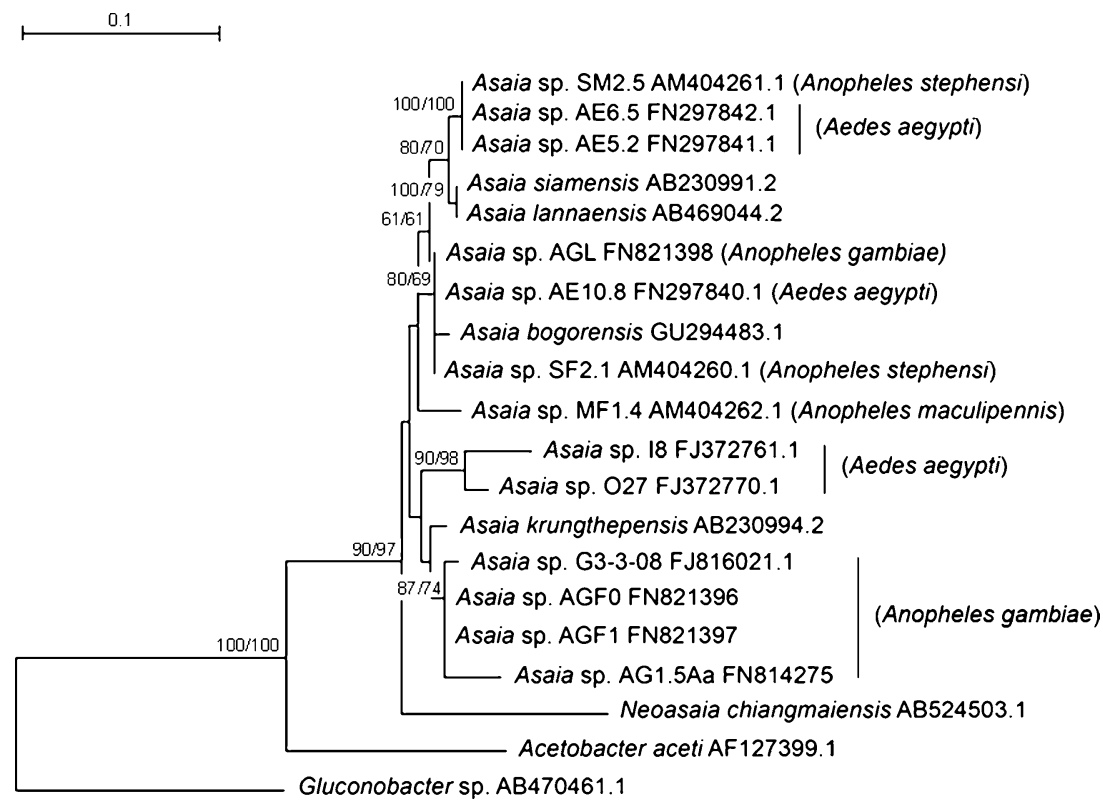


Table 2 Summary of the colonization experiments of $A$. gambiae (UNICAM strain) with GFP- or DsRed-tagged Asaia sp.; mosquitoes located in three different cages $(\mathrm{A}, \mathrm{B}, \mathrm{C})$ were allowed to consume recombinant bacteria: cage $\mathrm{A} / \mathrm{AGF} 0(\mathrm{pHM} 2-g f p)$; cage $\mathrm{B} / \mathrm{SF} 2.1$ (pHM2-gfp); cage C/SF2.1(DsRed)
Number of colonized mosquitoes/total and overall percentage
Day of bacterial detection ${ }^{\mathrm{a}}$, number of colonized mosquitoes/total and relative percentage

First detection Last detection

\begin{tabular}{|c|c|c|c|}
\hline \multicolumn{4}{|l|}{ Cage A: AGF0(pHM2-gfp) } \\
\hline Guts & $74 / 74(100 \%)$ & 2nd day 7/7 (100\%) & 20th day $9 / 9(100 \%)$ \\
\hline Salivary glands $q$ & $1 / 34(2.9 \%)$ & 2nd day $1 / 9(11.1 \%)$ & $=$ \\
\hline Reproductive organs + & $5 / 36(13.9 \%)$ & 2nd day $1 / 5(20 \%)$ & 20 th day $2 / 9(22.2 \%)$ \\
\hline Reproductive organs $\hat{\sigma}$ & $7 / 37(18.9 \%)$ & 6 th day $1 / 4(25 \%)$ & 20th day $2 / 10(20 \%)$ \\
\hline \multicolumn{4}{|l|}{ Cage B: SF2.1(pHM2-gfp) } \\
\hline Guts & $50 / 72(69.5 \%)$ & 2nd day $7 / 7(100 \%)$ & 20th day $4 / 13(30.7 \%)$ \\
\hline Salivary glands $q$ & $4 / 35(11.5 \%)$ & 4th day $3 / 6(50 \%)$ & 14 th day $1 / 5(20 \%)$ \\
\hline Reproductive organs $q$ & $9 / 36(25 \%)$ & 2nd day $2 / 5(40 \%)$ & $12^{\text {th }}$ day $1 / 4(25 \%)$ \\
\hline Reproductive organs $\hat{\jmath}$ & $0 / 35(0 \%)$ & No detection & No detection \\
\hline \multicolumn{4}{|l|}{ Cage C: SF2.1(DsRed) } \\
\hline Guts & $22 / 22(100 \%)$ & 2nd day 4/4 (100\%) & 20th day $4 / 4(100 \%)$ \\
\hline Salivary glands $q$ & $4 / 8(50 \%)$ & 2 nd day $1 / 2(50 \%)$ & 14 th day $1 / 2(50 \%)$ \\
\hline Reproductive organs $q$ & $8 / 12(66.7 \%)$ & 2 nd day $2 / 3(66.7 \%)$ & 20 th day $2 / 4(50 \%)$ \\
\hline Reproductive organs $\hat{\sigma}$ & $6 / 10(60 \%)$ & 2nd day $2 / 3(66.7 \%)$ & 16th day $2 / 3(66.7 \%)$ \\
\hline
\end{tabular}

${ }^{\text {a }}$ Fluorescence microscopy analysis of tissues for recombinant bacteria detection has been performed at different intervals from the 2 nd to the 20 th day postmosquitoes colonization by tagged-Asaia supplemented diet. The first and the last bacterial detection disaggregated data are reported

the guts (74 individuals analyzed) were abundantly colonized with GFP-tagged fluorescent bacteria (Fig. 3). Colonization was rarely observed in female salivary glands (one out 34 individuals analyzed) and in male (seven out 37 individuals analyzed) and female (five out 36 individuals analyzed) reproductive organs (Table 2 a). With respect to strain Asaia sp. SF2.1(pHM2-gfp) a duplicate colonization experiment was run following the same scheme adopted for

Figure 3 Gut colonization of $A$. gambiae (UNICAM strain) by GFP-tagged Asaia AGF0 (pHM2-gfp). Phase contrast (a) and fluorescence (b) microscope images of terminal portions of the midgut of a female mosquito; malpighian tubules are visible (a). Magnification of image B (c) and ventral diverticulum (d) showing high concentrations of GFP-tagged Asaia
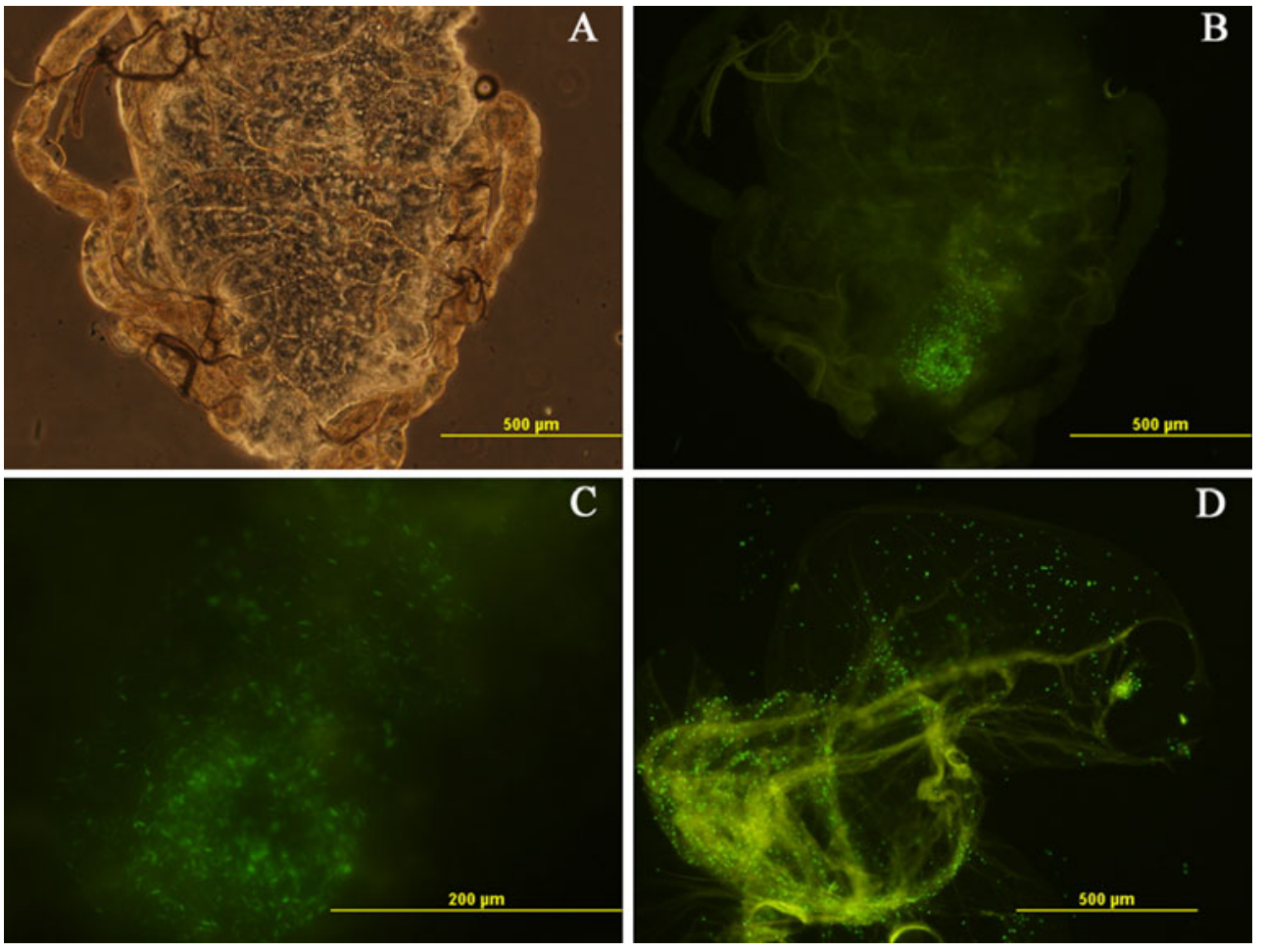
strain AGF0(pHM2-gfp). Colonization was achieved in guts (fifty out 72 individuals analyzed), in female salivary glands (four out 35 individuals analyzed) and in female reproductive organs (nine out 36 individuals analyzed), while no colonization was detected in male reproductive organs (Table 2 b). A further set of colonization experiments was performed with the red fluorescent strain Asaia sp. SF2.1 (DsRed) which stably retains the DsRed cassette without selective antibiotic pressure [4], supplemented into the sugar

Figure 4 Localization of Eubacteria and Asaia in the eggs of $A$. gambiae (UNICAM strain) and FISH control for the Eub and Asaia probes (both Asaia1. FCy3 and Asaia2. FCy3). Phase-contrast micrographs of A. gambiae eggs $(\mathbf{a}, \mathbf{d}, \mathbf{g}, \mathbf{l})$ and CLSM images of the same eggs after hybridization with the FITC-labelled EUB338 probe targeting Eubacteria $(\mathbf{b}, \mathbf{h})$ and the Cy3-labelled Asaia probes (c, f). Probes specificity has been verified by hybridization with the Cy3-labelled Asaia probes (f) in the absence of the FITC-labelled EUB338 probe targeting Eubacteria (e) and by hybridization with the FITClabelled probe targeting Eubacteria (h) in the absence of the Cy3-labelled Asaia probes (i). Control by RNase treatment before hybridisation with the FITC-labelled EUB338 probe targeting Eubacteria and the Cy3-labelled Asaia probes (m, n). Bar corresponds to $120 \mu \mathrm{m}$
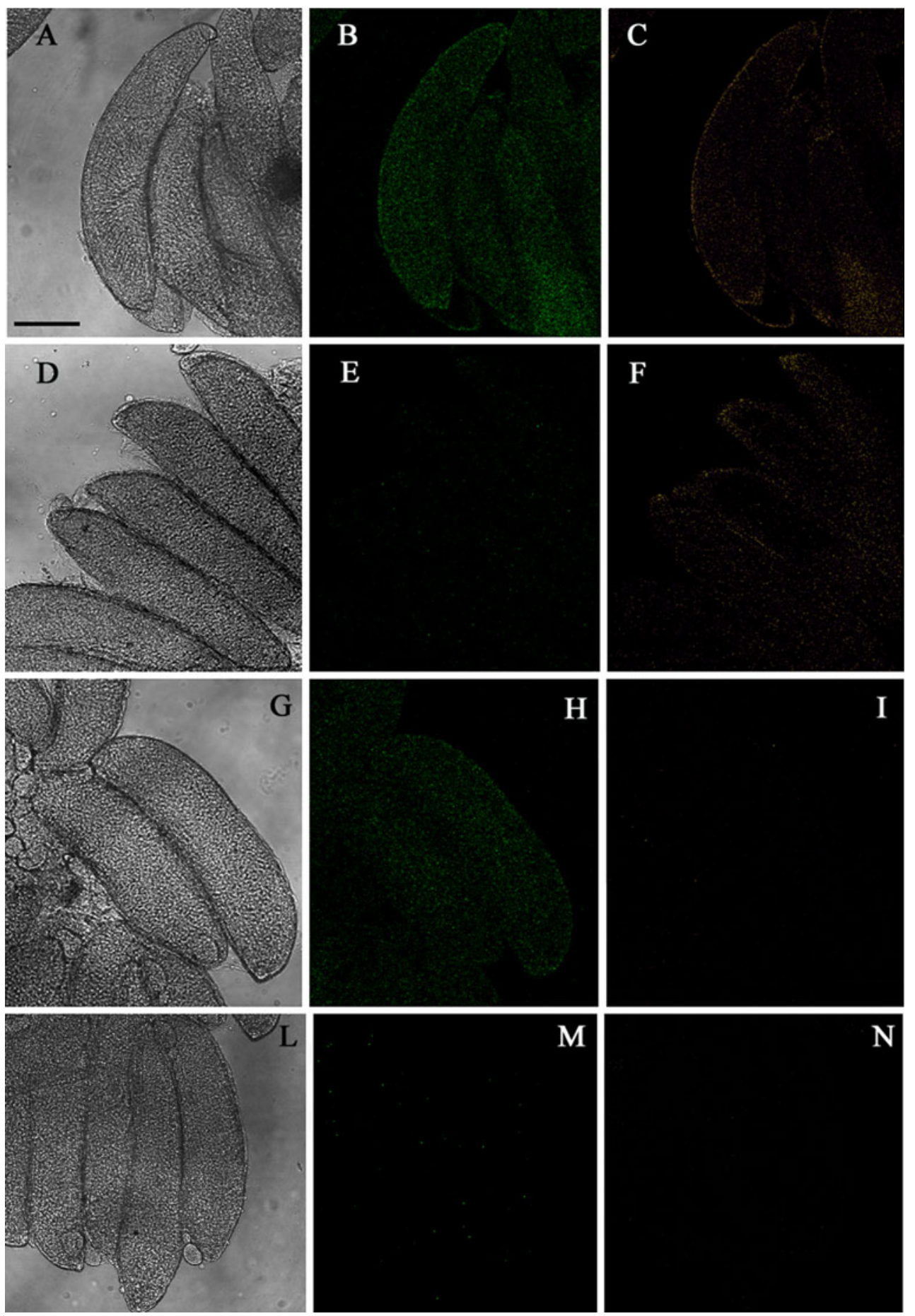
sampling period. In summary, all of the colonization experiments demonstrated a strong capacity of Asaia to invade the guts of the mosquitoes (with an efficiency up to $100 \%$ ); reproductive organs and salivary glands were also colonized, even though with fewer efficiency and with differences among the used strains.

Egg-Smearing-Mediated-Vertical Transmission Route of Asaia

The detection of Asaia in the reproductive organs and throughout the developmental stages of $A$. gambiae strongly indicates that these bacteria are vertically trans- mitted from parents to offspring in this species, as previously reported in $A$. stephensi $[4,9]$. Considering the importance of vertical transmission for the preservation and evolution of symbiotic relationships, we initiated an investigation in A. gambiae to uncover the mechanisms that ensure the transfer of Asaia from mother to offspring. We performed a FISH-based analysis to check for the localization of naturally occurring Asaia in the eggs isolated from ovaries of gravid mosquitoes (UNICAM strain) (Figs. 4, 5 and 6). Asaia was detected at the very periphery of the mosquito eggs (Fig. 4). To prove that signal was not due to autofluorescence, we included both positive (EUB338 probe targeting Eubacteria) and negative
Figure 5 Localization of Eubacteria and Asaia in the eggs of $A$. gambiae (UNICAM strain) at high magnification. A portion of the ovary reported as phasecontrast micrographs $(\mathbf{a}, \mathbf{b})$ has been stained with DAPI (c) and observed by CLSM after hybridization with the FITClabelled EUB338 probe targeting Eubacteria (d) and the Cy3-labelled Asaia probes (both Asaia1.FCy3 and Asaia2. FCy3) (f). Bacteria distribution has been verified by merging CLSM images with DAPI staining as shown in e and $\mathbf{g}$, respectively, for the Eubacteria and Asaia probes. Bar corresponds to $30 \mu \mathrm{m}$
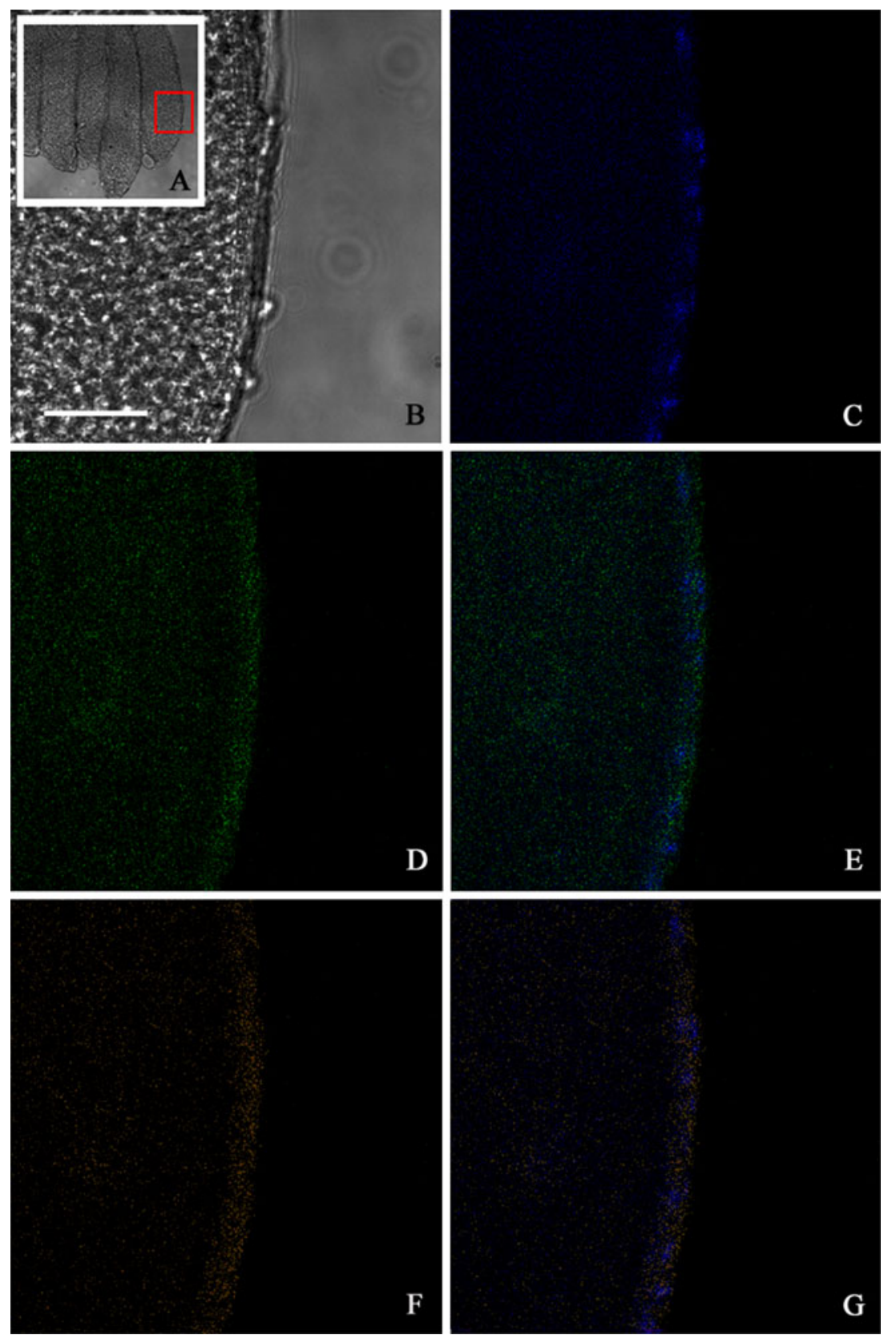
controls by hybridization with Cy3-tagged Asaia-specific probes (Fig. 4f) in the absence of the probe EUB338 (Fig. $4 \mathrm{e}$ ) and vice versa (Fig. $4 \mathrm{~h}$ and i, respectively). FISH micrographs of DAPI-stained bacterial DNA merged with signal from the Eubacteria- or Asaia-probes acquired at high magnification have been also provided (Fig. 5e and g, respectively). FISH hybridisation after RNase treatment provided evidence that the oligonucleotide probes did not unspecifically target cell components (Fig. $4 \mathrm{~m}$ and $\mathrm{n}$ ). Qualitative and quantitative evaluation of the distribution of Asaia at the periphery of the eggs indicate that the most of the bacteria are actually at the surface of the eggs (Fig. 6).

\section{Discussion}

The experimental results presented here indicate that Asaia is widely distributed in $A$. gambiae and recombinant strains showing the same colonization pattern and vertical transmission dynamics previously described in other mosquitoes as A. stephensi [9] and Ae. aegypti [3]. This suggests that Asaia is not restricted to certain mosquito species but can be generally widespread in many vector species.

Our findings go beyond the mere identification of Asaia in A. gambiae representing the first broad observation of these bacteria in the wild and supporting the proposal for the application of this microorganism as a carrier of antiplasmodial factors, as in for example a paratransgenic approach for malaria control in Afro-tropical regions where A. gambiae is the major mosquito vector.
Several features support this proposal: (1) the retrieved pattern of localization indicates that Asaia occurs in the same locales important for the parasite development (midgut and salivary glands) and for transmission to the progeny (reproductive organs) as already demonstrated for A. stephensi. (2) Asaia shows a stable association with preand adult mosquitoes as witnessed by its presence in all the samples analyzed belonging to different generations. (3) Colonization experiments reveal that different modified strains of Asaia, acquired by mosquitoes via consumption, are able to occupy in large amounts the midgut of all the recipient mosquitoes treated, as well as, salivary glands and reproductive organs where differences in colonization efficiency are probably due to both direct effects of the antibiotic and different ability of the employed strains to colonize mosquito's organs. (4) Asaia has now been isolated from both laboratory reared and field collected $A$. gambiae and previously from A. stephensi and A. maculipennis [9], three species of malaria vectors which are present in quite distant regions of the world, suggesting that the introduction of modified Asaia into mosquito populations would bypass genetic barriers of reproductively isolated mosquito populations occurring in endemic malaria regions which often interfere with the success of vector control strategies [11].

Finally, we have provided evidence which suggest that the transfer of Asaia from parents to offspring could be mediated by a mechanism of egg-smearing, as previously proposed for the leafhopper Scaphoideus titanus [3]. Indeed, TEM observations failed to detect intracellular
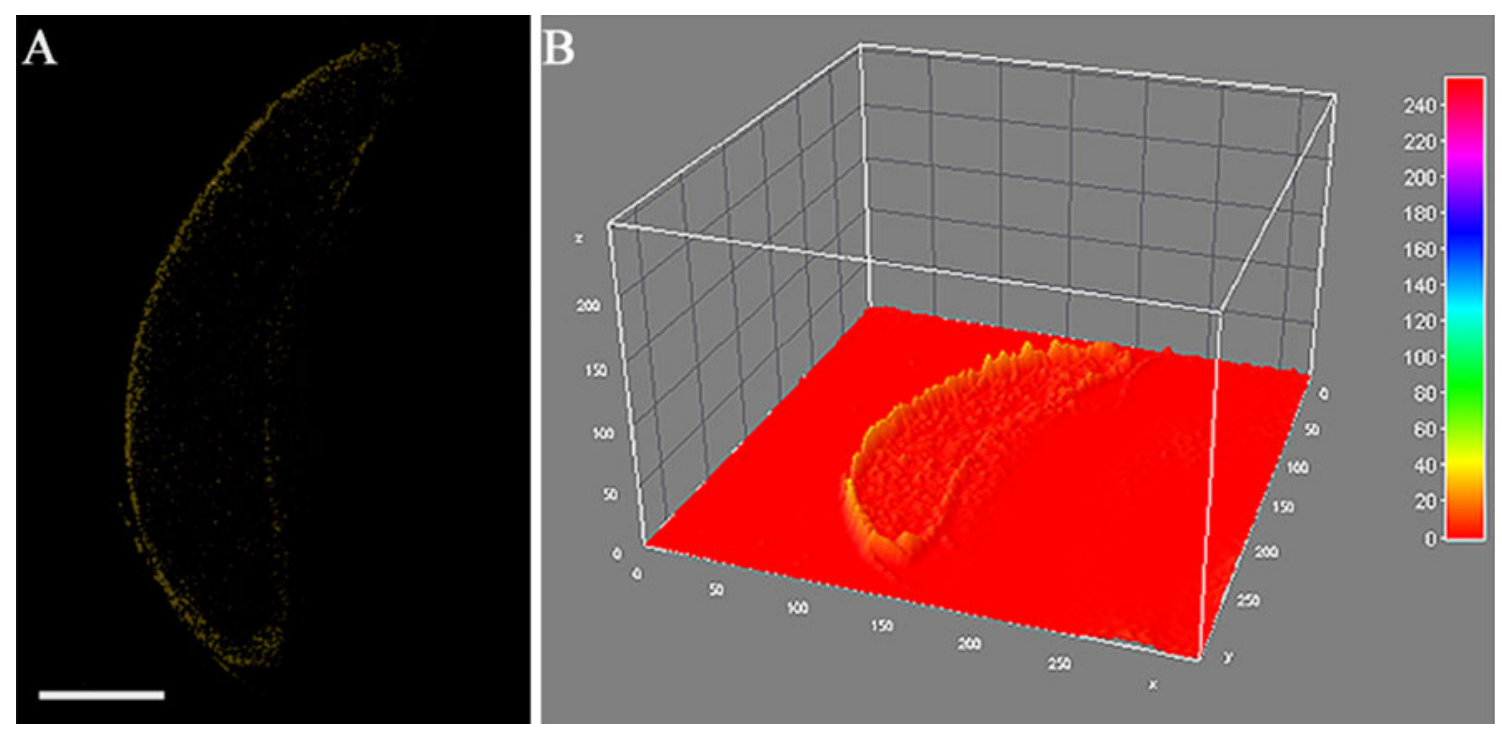

Figure 6 Localization of Asaia in the eggs of Anopheles gambiae (UNICAM strain). (a) CLSM image of an egg after hybridization with the Cy3-labelled Asaia probes. (b) A three-dimensional (3D) reconstruction of the Cy3-labelled egg using the Interactive $3 \mathrm{D}$ Surface Plot plugin of ImageJ shows that the fluorescence is concentrated at the periphery of the egg 
bacteria inside pre-vitellogenic eggs of both $A$. gambiae and $A$. stephensi (Sacchi L., unpublished observations). This would suggest that the entrance of the bacteria occurs later during oogenesis or successive development. On the other hand, the pictures demonstrating that Asaia is smeared on the surface of the egg are really convincing for S. titanus [3], another insect model in which Asaia has not been observed in pre-vitellogenic eggs (Sacchi L., unpublished observations). Taken together, all of the available information are thus coherent in indicating that Asaia transmission to the progeny is also egg-mediated. We could thus hypothesize that these extracellular symbionts are smeared onto the eggs, and then consumed by the hatching larvae, as reported for other insects $[6,13]$.

Acknowledgments The project was supported by the Firb-Ideas (grant RBID082MLZ) and Prin 2007 (grant 2007PK2HB7_002), both from the Italian Ministry of University and Research (MIUR), and by the EU-FP7 Capacities-Infrastructure 2008 (grant 228421) to G.F.

E.C. and P.R. benefited of travel grant from the COST Action FA0701 and support from the Swedish Research Council to I.F. and A.K.

I.R. was funded by "Compagnia di San Paolo" in the context of the Italian Malaria Network.

We thank Kristina Havas for English revision of the manuscript.

\section{References}

1. Beier JC (1998) Malaria parasite development in mosquitoes. Annu Rev Entomol 43:519-543, Review

2. Breman JG, Alilio MS, Mills A (2004) Conquering the intolerable burden of malaria: what's new, what's needed: a summary. Am J Trop Med Hyg 71:1-15

3. Crotti E, Damiani C, Pajoro M, Gonella E, Rizzi A, Ricci I, Negri I, Scuppa P, Rossi P, Ballarini P, Raddadi N, Marzorati M, Sacchi L, Clementi E, Genchi M, Mandrioli M, Bandi C, Favia G, Alma A, Daffonchio D (2009) Asaia, a versatile acetic acid bacterial symbiont, capable of cross-colonizing insects of phylogeneticallydistant genera and orders. Environ Microbiol 11:3252-3264

4. Damiani C, Ricci I, Crotti E, Rossi P, Rizzi A, Scuppa P, Esposito F, Bandi C, Daffonchio D, Favia G (2008) Paternal transmission of symbiotic bacteria in malaria vectors. Curr Biol 18:1087-1088

5. Daims H, Brühl A, Amann R, Schleifer KH, Wagner M (1999) The domain-specific probe EUB338 is insufficient for the detection of all Bacteria: development and evaluation of a more comprehensive probe set. Syst Appl Microbiol 22:434-444

6. Douglas AE (1989) Mycetocyte symbiosis in insects. Biol Rev Camb Philos Soc 64:409-434, Review

7. Edgar RC (2004) MUSCLE: multiple sequence alignment with high accuracy and high throughput. Nucleic Acids Res 32:1792-1797
8. Favia G, Dimopoulos G, della Torre A, Touré YT, Coluzzi M, Louis C (1994) Polymorphisms detected by random PCR distinguish between different chromosomal forms of Anopheles gambiae. Proc Natl Acad Sci USA 91:10315-10319

9. Favia G, Ricci I, Damiani C, Raddadi N, Crotti E, Marzorati M, Rizzi A, Urso R, Brusetti L, Borin S, Mora D, Scuppa P, Pasqualini L, Clementi E, Genchi M, Corona S, Negri I, Grandi G, Alma A, Kramer L, Esposito F, Bandi C, Sacchi L, Daffonchio D (2007) Bacteria of the genus Asaia stably associate with Anopheles stephensi, an Asian malarial mosquito vector. Proc Natl Acad Sci USA 104:9047-9051

10. Favia G, Ricci I, Marzorati M, Negri I, Alma A, Sacchi L, Bandi C, Daffonchio D (2008) Bacteria of the genus Asaia: a potential paratransgenic weapon against malaria. Adv Exp Med Biol 627:49-59

11. Fontenille D, Cohuet A, Awono-Ambene P, Kengne P, AntonioNkondjio C, Wondji C, Simard F (2005) Malaria vectors: from the field to genetics, Research in Africa. Rev Epidemiol Sante Publique 53:283-290

12. Ito J, Ghosh A, Moreira AL, Wimmer EA, Jacobs-Lorena M (2002) Transgenic anopheline mosquitoes impaired in transmission of a malaria parasite. Nature 417:452-455

13. Iverson KL, Bromel MC, Anderson AW, Freeman TP (1984) Bacterial symbiont in the sugar beet root maggot, Tetanops myopaeformis. Appl Environ Microbiol 47:22-27

14. Jacobs-Lorena M (2003) Interrupting malaria transmission by genetic manipulation of anopheline mosquitoes. J Vector Borne Dis $40: 73-77$

15. James AA (2003) Blocking malaria parasite invasion of mosquito salivary glands. J Exp Biol 206:3817-3821

16. Knols BG, Bossin HC, Mukabana WR, Robinson AS (2007) Transgenic mosquitoes and the fight against malaria: managing technology push in a turbulent GMO world. Am J Trop Med Hyg $77: 232-242$

17. Mølbak L, Molin S, Kroer N (2007) Root growth and exudate production define the frequency of horizontal plasmid transfer in the Rhizosphere. FEMS Microbiol Ecol 59:167-176

18. Riehle MA, Jacobs-Lorena M (2005) Using bacteria to express and display anti-parasite molecules in mosquitoes: current and future strategies. Insect Biochem Mol Biol 35:699-707, Review

19. Riehle MA, Moreira CK, Lampe D, Lauzon C, Jacobs-Lorena M (2007) Using bacteria to express and display anti-Plasmodium molecules in the mosquito midgut. Int J Parasitol 37:595-603

20. Sacchi L, Genchi M, Clementi E, Bigliardi E, Avanzati AM, Pajoro M, Negri I, Marzorati M, Gonella E, Alma A, Daffonchio D, Bandi C (2008) Multiple symbiosis in the leafhopper Scaphoideus titanus (Hemiptera: Cicadellidae). Tissue Cell 40:231-242

21. Van de Peer Y, De Wachter R (1997) Construction of evolutionary distance trees with TREECON for Windows: accounting for variation in nucleotide substitution rate among sites. Comput Appl Biosci 13:227-230

22. Yamada Y, Katsura K, Kawasaki H, Widyastuti Y, Saono S, Seki T, Uchimura T, Komagata K (2000) Asaia bogorensis gen. nov., sp. nov., an unusual acetic acid bacterium in the alphaProteobacteria. Int J Syst Evol Microbiol 50:823-829 